\title{
Cerebrospinal fluid S-adenosylmethionine in depression and dementia: effects of treatment with parenteral and oral S-adenosylmethionine
}

\author{
T Bottiglieri, P Godfrey, T Flynn, M W P Carney, B K Toone, E H Reynolds
}

\begin{abstract}
Cerebrospinal fluid (CSF) S-adenosylmethionine (SAM) levels were significantly lower in severely depressed patients than in a neurological control group. The administration of SAM either intravenously or orally is associated with a significant rise of CSF SAM, indicating that it crosses the blood-brain barrier in humans. These observations provide a rational basis for the antidepressant effect of SAM, which has been confirmed in several countries. CSF SAM levels were low in a group of patients with Alzheimer's dementia suggesting a possible disturbance of methylation in such patients and the need for trials of SAM treatment.
\end{abstract}

S-adenosylmethionine (SAM) is the sole methyl donor in many important methylation reactions in the nervous system, involving neurotransmitters, amines and polyamines, membrane phospholipids, proteins and nucleoproteins. ${ }^{1}$ SAM is intimately linked to the folate cycle and vitamin B12 metabolism, which provides many of the methyl groups required for the SAM mediated methylation reactions, via the synthesis of methionine by the enzyme methionine synthetase. ${ }^{2}$

In Italy clinical trials on severely depressed patients have suggested that SAM has antidepressant properties ${ }^{23}$ and this has now been supported by studies in several countries. $^{4-7}$ There is also evidence that severe folate deficiency may lead, amongst other complications, to depression. ${ }^{8-11}$ Both sets of observations suggest that methylation reactions are important in the regulation of mood and may be implicated in some affective disorders. ${ }^{210}$

During our placebo controlled investigations of SAM in depression ${ }^{5}$ we have included examinations of cerebrospinal fluid (CSF) SAM before and after treatment. For these

Table 1 CSF SAM in neurological controls, depression and dementia

\begin{tabular}{lllll}
\hline & $\begin{array}{l}\text { Neurological } \\
\text { controls }\end{array}$ & $\begin{array}{l}\text { Multiple } \\
\text { sclerosis }\end{array}$ & Depression & $\begin{array}{l}\text { Alzheimer's } \\
\text { dementia }\end{array}$ \\
\hline $\mathrm{N}$ & 13 & 16 & 35 & 9 \\
Sex $(\mathrm{M} / \mathrm{F})$ & $6 / 7$ & $6 / 10$ & $13 / 22$ & $6 / 3$ \\
Age (years) & $49 \cdot 7(16 \cdot 4)$ & $41 \cdot 1(9 \cdot 7)$ & $52 \cdot 3(16 \cdot 6)$ & $60 \cdot 3(5 \cdot 5)$ \\
CSF SAM & $64.5(11 \cdot 9)$ & $78 \cdot 0(23 \cdot 9)$ & $56 \cdot 0(13 \cdot 8)^{\star}$ & $36 \cdot 1(13 \cdot 8) \dagger$ \\
\hline Values indicate mean (SD) & & \\
Comparison with neurological controls using the $t$ test: \\
$\star_{t=2 \cdot 10, \mathrm{p}=0.041}$ \\
$t t=4 \cdot 76, \mathrm{p}=0.0001$
\end{tabular}

studies SAM was administered by the intravenous route daily for 14 days. While examining CSF SAM in a control group of patients with neurological disorders we noted that the lowest values were seen in patients with dementia. ${ }^{12}$ Recently an oral formulation of SAM has become available, ${ }^{13}$ and this has enabled us to examine CSF SAM in a small group of demented patients before and after oral SAM treatment for several months.

\section{Patients and methods}

CSF SAM was examined in a) neurological control groups comprising 16 patients with clinically definite multiple sclerosis, three with motor neuron disease, seven with unexplained peripheral neuropathy, one with benign intracranial hypertension, one with narcolepsy and one with encephalomyelitis; b) 35 patients with severe depression according to Feighner criteria, all of whom scored 20 or more points on the Hamilton Rating Scale; c) nine patients with Alzheimer's dementia according to the DSM-3-R classification. The age and sex of the patient groups are summarised in table 1.

Thirty of the depressed patients participated in a double blind study of treatment either with intravenous SAM 200 mgs daily or intravenous placebo daily for 14 days. Sixteen patients received SAM and 14 placebo. Cerebrospinal fluid SAM was re-examined at the end of the trial. In three patients from each group the CSF was taken two hours after the last injection, and in the remainder 24 hours after the last injection.

Four patients with Alzheimer's dementia were treated openly with oral SAM 1200 mgs daily (400 mgs three times a day). CSF SAM was re-examined after treatment periods which ranged from four to eight months.

Intravenous (200 mgs) and oral (400 mgs enteric coated tablets) SAM was supplied by Bioresearch, Milan, Italy. CSF and plasma SAM were determined in perchloric acid extracts by a double isotope enzymatic assay. ${ }^{14}$ We do not know if there is a rostro-caudal gradient in the CSF for SAM but lumbar puncture was always undertaken after overnight fasting and rest and the SAM assay undertaken on the first $5 \mathrm{mls}$ of fluid.

Results

We found no sex differences for CSF SAM. The CSF SAM levels in the different patient groups are shown in table 1 . The highest values 
Table 2 The effect of IV SAM treatment on CSF SAM in depressed patients

\begin{tabular}{llll}
\hline & Baseline & $\begin{array}{l}\text { Two hours post } \\
\text { treatment }\end{array}$ & $\begin{array}{l}\text { 24 hours post } \\
\text { treatment }\end{array}$ \\
\hline Placebo & $53 \cdot 8(21 \cdot 2)$ & $55 \cdot 8(23 \cdot 6)$ & $57 \cdot 4(14 \cdot 4)$ \\
Number of patients & 14 & 3 & 11 \\
IV SAM 200 mg & $54 \cdot 4(13 \cdot 7)$ & $91 \cdot 0(14 \cdot 3) \dagger$ & $64 \cdot 0(12 \cdot 6)^{\star}$ \\
Number of patients & 16 & 3 & 13 \\
\hline
\end{tabular}

Depressed patients were treated for 14 days and post samples taken on the last day of treatment. Values indicate mean $(\mathrm{SD})$ in $\mathrm{ng} / \mathrm{ml}$. ANOVA: ${ }^{\mathrm{p}}<0.02,+\mathrm{p}<0.001$

Table 3 The effect of oral SAM treatment on plasma and CSF SAM in patients with dementia

\begin{tabular}{lcl}
\hline & Plasma SAM & CSF SAM \\
\hline Pre treatment $(\mathrm{n}=4)$ & $33.6(4 \cdot 5)$ & $35 \cdot 5(13.8)$ \\
Post treatment $(\mathrm{n}=4)$ & $135.4(68 \cdot 3)^{\star}$ & $66.0(10.9) \dagger$
\end{tabular}

Post samples were taken 1.5-5.0 hours after the last oral dose. Values indicate mean (SD) in $\mathrm{ng} / \mathrm{ml}$ paired $t$-test, ${ }^{\star} \mathrm{t}=6.33$, $\mathrm{p}=0.008 ; \mathrm{tt}=7 \cdot 24, \mathrm{p}=0.0054$.

were seen in patients with multiple sclerosis. Although for this control subgroup the increase in CSF SAM compared with the remaining control subgroup was not statistically significant we have documented the two control subgroups separately. Statistically significant lowering of CSF SAM was found in the groups with depression and with Alzheimer's dementia compared with the neurological control group and with the two individual subgroups; the lowest levels occurring in the Alzheimer's group (table 1).

The administration of intravenous SAM $200 \mathrm{mgs}$ daily for two weeks to depressed patients is associated with a significant rise in CSF SAM, a phenomenon which is not seen after two weeks treatment with placebo (table 2). Higher values for CSF SAM are seen two hours after the last injection ( $67 \%$ increase) than 24 hours after the last injection (19\% increase) (table 2). Oral SAM treatment (1200 mgs daily) for four to eight months was associated with a significant increase $(62.5 \%)$ in CSF SAM in patients with Alzheimer's dementia (table 3 ). The post-treatment values were within the neurological control range (tables 1 and 3 ).

\section{Discussion}

S-adenosylmethionine is a ubiquitous metabolite which, when administered intravenously in normal subjects and patients in single doses varying from 100 to $500 \mathrm{mgs}$, is rapidly eliminated from the circulation with a half-life of about 90 minutes. ${ }^{15}$ We have previously shown that following single oral doses of 400 to $1000 \mathrm{mgs}$ in normal subjects peak plasma levels are achieved between two and six hours and SAM is thereafter rapidly metabolised from the circulation. ${ }^{16}$

In this study we have shown that following intravenous or oral administration SAM crosses the blood-brain barrier leading to a rise in CSF SAM (tables 2 and 3). In the placebo controlled study in depressed patients CSF SAM was re-examined either two or 24 hours after the last of 14 daily injections. In keeping with the pharmacokinetics of SAM in blood the greatest rise $(67 \%)$ in CSF was seen at two hours, but even at 24 hours there was a $19 \%$ elevation. No rise was seen with placebo at two or 24 hours. After oral administration of SAM continuously for four to eight months in demented patients CSF SAM rose by nearly two-thirds from baseline levels into the control range.

The therapeutic potential of SAM in some forms of depression, especially endogenous depression, has now been reported in several countries. ${ }^{2-7}$ Until recently the trials have utilised parenteral SAM, but longer term oral SAM studies are now showing promising results. ${ }^{131718}$ SAM is remarkably free from side effects, the main problem being the precipitation of hypomania or mania in some patients with bipolar depression (which can occur with any antidepressant), and is further evidence of its efficacy. ${ }^{18}$

We have demonstrated in a large series of severely depressed patients that CSF SAM is significantly lower than in two neurological control groups (table 1). Although in the subgroup of multiple sclerotic patients the CSF SAM is not significantly higher than the other neurological control patients the trend is such that we thought it appropriate to keep the subgroups separate, especially as some patients have been described recently with multiple sclerosis associated with abnormalities of vitamin B12 metabolism. ${ }^{19}$ The low level of CSF SAM in some patients with depression suggests that there may be an underlying disturbance in methylation within the nervous system and provides a rational basis for the therapeutic use of SAM, at least in this subgroup. We have recently confirmed other evidence of abnormal methylation in depression in the form of reduced erythrocyte methionine adenosyltransferase (MAT) activity, ${ }^{16}$ as was earlier reported by Alarcon et al. ${ }^{20} \mathrm{MAT}$ is the enzyme responsible for the synthesis of SAM from methionine.

During our study of other neurological diagnostic groups it became apparent that patients with Alzheimer's dementia had remarkably low levels of CSF SAM, significantly lower than in depression (table 1). This suggests that disturbance in methylation in the brain may also be important in some forms of dementia. The administration of oral SAM to four of our patients was associated with an increase in CSF SAM to within the control range (table 3 ) and was accompanied by an improvement in mood and cognitive measures such as to justify further controlled trials of SAM in this clinical situation. ${ }^{21}$ The potential importance of such studies has recently been indicated by Holliday $^{22}$ who has reviewed the role of DNA methylation in "epigenetic" mechanisms which influence the long term functioning of tissues. He summarises evidence to support the view that the gradual loss of methyl groups derived from SAM could contribute to ageing.

\footnotetext{
1 Baldessarini RJ. Neuropharmacology of S-adenosylmeth-

ionine. Am J Med 1987;83(Suppl 5a):95-103.
Reynolds EH, Stramentinoli G. Folic acid, S-adenosylmethionine and affective disorder. Psychol Med 1983; 13:705-10

3 Agnoli A, Andreoli V, Casacchia M, et al. Effect of Sadenosylmethionine (SAMe) upon depressive symptoms.
} 
$J$ Psychiatr Res 1976;13:43-54.

4 Kufferle B, Grunberger J. Early clinical double blind study with S-adenosyl-L-methionine; a new potential antidepressant. In: Costa E, Racagni G, eds. Typical and atypical antidepressants: clinical practice. New York: Raven Press, 1982:175-180.

5 Carney MWP, Edeh J, Bottiglieri T, et al. Affective illness and S-adenosylmethionine. Clin neuropharmacol 1986; 9:379-85.

6 Janicak PG, Lipinski J, Davis JM, et al. S-adenosylmethionine in depression: a literature review and preliminary nine in depression: a literature review and pre

7 Bell KM, Plon L, Bunney WE, Potkin G. S-adenosylmeth ionine treatment of depression: a controlled clinical trial Am J Psychiatr 1988;145:1110-14.

8 Reynolds EH. Effects of folic acid on the mental state and fitfrequency of drug-treated epileptic patients. Lancet 1967 i: $1086-8$.

9 Carney MWP. Serum folate values in 423 psychiatric patients. Br Med J 1967;4:512-16.

10 Reynolds EH, Carney MWP, Toone BK. Methylation and mood. Lancet 1984;ii:196-7.

11 Reynolds EH. Folic acid and neuropsychiatry. Farmacia Terapia 1985;2:163-5.

12 Bottiglieri T, Carney MWP, Edeh J, et al. A biochemical study of depressed patients receiving $S$-adenosylmethionine (SAMe). In: Borchardt RT, Creveling CR, Ueland nine (SAMe). In: Borchardt RT, Creveling CR, Ueland PM, eds. Biological methylation and drug

13 Carney MWP, Chary TKN, Bottiglieri T, et al. The switch mechanism in affective illness and oral S-adenosylmethionine (SAM). Br J Psychiatr 1987;150:724.

14 Guilidori P, Stramentinoli G. A radioenzymatic method for S-adenosylmethionine determination in biological fluids. Analytical biochem 1984;137:217-20.

15 Guilidori P, Cortellaro M, Moreo G, et al. Pharmacokinetics of $\mathrm{S}$-adenosylmethionine in healthy volunteers. Eur J Clin Pharmacol 1984;27:110-21.

16 Bottiglieri T, Chary TK, Laundy $M$, et al. Transmethyla tion in depression. Alabama J Med Sciences 1988;25: 296-301.

17 Rossenbaum JF, Fava M, Falk WE, et al. An open-label pilot study of oral S-Adenosyl-L-Methionine in major depression. Alabama J Med Sciences 1988;25:301-6.

18 Carney MWP, Chary TKN, Bottiglieri T, Reynolds EH. The switch mechanism and the bipolar/unipola dichotomy. Br J Psychiatr 1989;154:48-51.

19 Reynolds EH. Linnell JC. Vitamin B12 deficiency, demyelination and multiple sclerosis. Lancet 1987;2:920.

20 Alarcon RD, Tolbert LC, Monti JA, et al. One carbon metabolism disturbances in affective disorders. $J$ Affect Dis 1985;9:297-301.

21 Reynolds EH, Godfrey P, Bottiglieri T, et al. S-adenosylmethionine and Alzheimer's disease. Neurology 1989; 39(Suppl 1):397.

22 Holliday $R$ A different kind of inheritance: the methylation of DNA may be a major epigenetic mechanism by which gene-activity patterns-as opposed to genes per se-are passed from one generation of cells to another during development. Scientific American 1989;260(6):40-8. 